\title{
Correction: eEF-2 kinase is a critical regulator of Warbrug effect through controlling PP2A-A synthesis
}

\author{
Y Cheng ${ }^{1,2} \cdot X$ Ren $^{2} \cdot Y Y_{\text {Yuan }}^{3} \cdot Y$ Shan $^{2} \cdot \mathrm{L} \mathrm{Li}^{1} \cdot \mathrm{X} \mathrm{Chen}^{1} \cdot \mathrm{L} \mathrm{Zhang}^{2} \cdot \mathrm{Y}$ Takahashi $^{2} \cdot \mathrm{J} \mathrm{W} \mathrm{Yang}^{2} \cdot \mathrm{B} \mathrm{Han}^{4} \cdot \mathrm{J} \mathrm{Liao}^{5} \cdot \mathrm{Y} \mathrm{Li}^{2} \cdot$

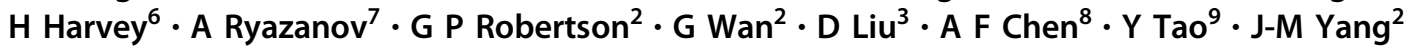

Published online: 4 July 2018

(c) Macmillan Publishers Limited, part of Springer Nature 2018

Correction to: Oncogene (2016) 35, 6293-6308; https:// doi.org/10.1038/onc.2016.166; published online 16 May 2016

In this article, the authors recently noticed that the tubulin blots in Figs. 2a and $6 a$ were inadvertently misplaced during the preparation of these figures due to their similarity. The amended versions of the figures are now shown below. The conclusions of this paper are not affected. The authors sincerely apologize for these errors.
Y Cheng

chengyan0677@163.com

J-M Yang

juy16@psu.edu

1 Department of Pharmacology, School of Pharmaceutical Sciences, Central South University, Changsha, China

2 Department of Pharmacology, The Penn State Hershey Cancer Institute, The Pennsylvania State University College of Medicine and Milton S Hershey Medical Center, Hershey, PA, USA

3 Department of Pharmaceutical Sciences, Washington State University College of Pharmacy, Spokane, WA, USA

4 Department of Pathology, The Penn State Hershey Cancer Institute, The Pennsylvania State University College of Medicine and Milton S Hershey Medical Center, Hershey, PA, USA
5 Division of Biostatistics and Bioinformatics, The Penn State Hershey Cancer Institute, The Pennsylvania State University College of Medicine and Milton S Hershey Medical Center, Hershey, PA, USA

6 Department of Medicine, The Penn State Hershey Cancer Institute, The Pennsylvania State University College of Medicine and Milton S Hershey Medical Center, Hershey, PA, USA

7 Department of Pharmacology, Robert wood Jonson Medical School, Rutgers University, Piscataway, NJ, USA

8 Center for Vascular and Translational Medicine, College of Pharmacy, and The Third Xiangya Hospital, Central South University, Changsha, Hunan, China

9 Cancer Research Institute, School of Basic Medicine, and Key Laboratory of Carcinogenesis and Cancer Invasion, Ministry of Education, Central South University, Changsha, China 


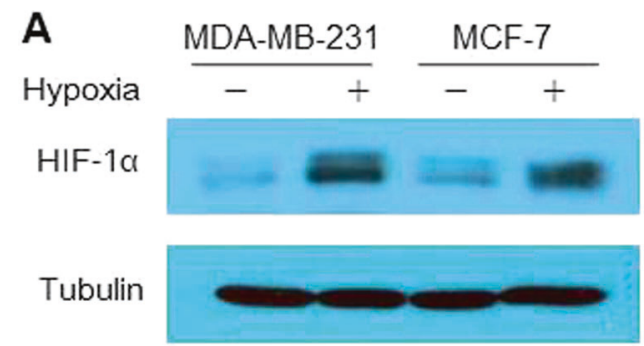

Fig. 2 a

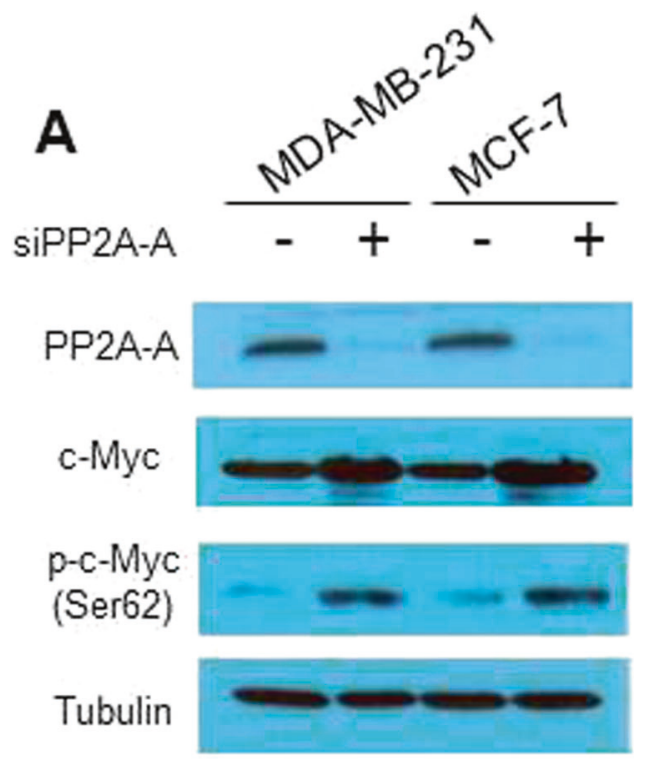

Fig. 6 a 